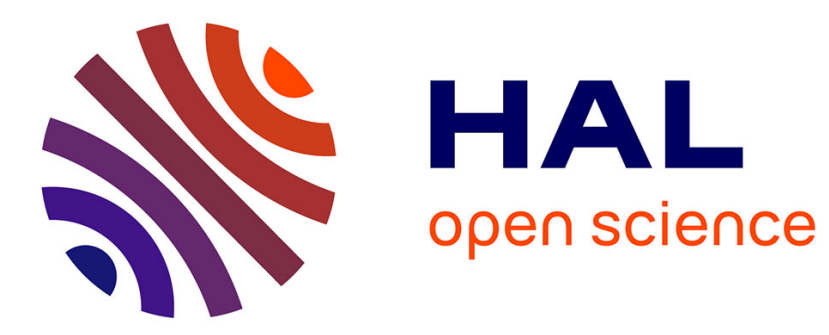

\title{
Multiple Sclerosis Lesions Evolution in Patients with Clinically Isolated Syndrome
}

Alessandro Crimi, Olivier Commowick, Jean-Christophe Ferré, Adil Maarouf, Gilles Edan, Christian Barillot

\section{- To cite this version:}

Alessandro Crimi, Olivier Commowick, Jean-Christophe Ferré, Adil Maarouf, Gilles Edan, et al.. Multiple Sclerosis Lesions Evolution in Patients with Clinically Isolated Syndrome. SPIE Medical Imaging 2013: Image Processing, Feb 2013, United States. pp.86690I-86690I-8. inserm-00781478

HAL Id: inserm-00781478 https://www.hal.inserm.fr/inserm-00781478

Submitted on 4 Jun 2013

HAL is a multi-disciplinary open access archive for the deposit and dissemination of scientific research documents, whether they are published or not. The documents may come from teaching and research institutions in France or abroad, or from public or private research centers.
L'archive ouverte pluridisciplinaire HAL, est destinée au dépôt et à la diffusion de documents scientifiques de niveau recherche, publiés ou non, émanant des établissements d'enseignement et de recherche français ou étrangers, des laboratoires publics ou privés. 


\title{
Multiple Sclerosis Lesions Evolution in Patients with Clinically Isolated Syndrome
}

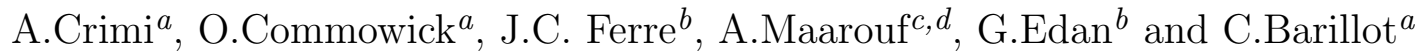 \\ ${ }^{a}$ INRIA, 263 Av. du G. Leclerc, Rennes, France; ${ }^{b}$ CHU, 2 Rue H. le Guilloux, Rennes, France; \\ ${ }^{c}$ Neurology Departement, University hospital Reims, France; \\ ${ }^{d}$ CRMBM, Aix-Marseille University, CNRS, Marseille, France;
}

\begin{abstract}
Multiple sclerosis (MS) is a disease with heterogeneous evolution among the patients. Some classifications have been carried out according to either the clinical course or the immunopathological profiles. Epidemiological data and imaging are showing that MS is a two-phase neurodegenerative inflammatory disease. At the early stage it is dominated by focal inflammation of the white matter (WM), and at a latter stage it is dominated by diffuse lesions of the grey matter and spinal cord. A Clinically Isolated Syndrome (CIS) is a first neurological episode caused by inflammation/demyelination in the central nervous system which may lead to MS. Few studies have been carried out so far about this initial stage. Better understanding of the disease at its onset will lead to a better discovery of pathogenic mechanisms, allowing suitable therapies at an early stage.

We propose a new data processing framework able to provide an early characterization of CIS patients according to lesion patterns, and more specifically according to the nature of the inflammatory patterns of these lesions. The method is based on a two layers classification. Initially, the spatio-temporal lesion patterns are classified using a tensor-like representation. The discovered lesion patterns are then used to identify group of patients and their correlation to 15 months follow-up total lesion loads (TLL), which is so far the only image-based figure that can potentially infer future evolution of the pathology.

We expect that the proposed framework can infer new prospective figures from the earliest imaging sign of MS since it can provide a classification of different types of lesion across patients.
\end{abstract}

Keywords: MS, clinically isolated syndrome, spectral clustering

\section{DESCRIPTION OF PURPOSE}

Multiple Sclerosis (MS) is an acquired inflammatory, demyelinating disease of the central nervous system. MS is a major cause of disability in young adults very common in the northern hemisphere. The natural evolution of MS is highly variable and it is still not fully understood, though its nature seems related to a two-phase autoimmune response $^{1}$. At the moment it is impossible to predict accurately the severity, nature and progression for each patient. The most known MS categorization is related to the disease course. In a Relapsing-remitting MS (RRMS) a patient experiences repetitively some symptoms for a short period of time and subsequently experiences no new signs of disease activity. More than half of RRMS patients eventually decline to a more chronic state called secondary-progressive MS (SPMS). SPMS is characterized by gradual neurological deterioration. If the disease progression is constant from the beginning, it is called primary-progressive MS (PPMS) ${ }^{1}$. Another classification is given by the so called Lassman patterns ${ }^{2}$, which are patterns related to the response of the immune system discovered at microscopic level from biopsies and autopsies of MS patients. No neat correlation between Lassman patterns, MRI analysis, and the clinical course classification has emerged so far.

In acute MS, macrophages act more strongly than T-cells ${ }^{3}$. Hence, studying the early deposits of inflamed macrophages using specific markers is expected to lead to a better understanding of the pathology and of its evolution. This can be done using a novel contrast agent, called Ultrasmall Superparamagnetic Iron Oxide (USPIO) which is sensitive to macrophages activity and using patients at the onset of the disease. USPIO

Further author information. E-mail: christian.barillot@inria.fr , Telephone: +33299842522

Medical Imaging 2013: Image Processing, edited by Sebastien Ourselin, David R. Haynor, Proc. of SPIE Vol. 8669, 86690I · C 2013 SPIE · CCC code: 1605-7422/13/\$18 doi: $10.1117 / 12.2006647$

Proc. of SPIE Vol. $8669866901-1$ 
shows different spatio-temporal patterns of enhancement compared to the traditional Gadolinium (Gd) due to its nature of highlighting macrophages ${ }^{4}$.

The MS onset can be studied on CIS patients, which are patients who experienced a first attack suggestive of demyelination, but do not fulfill yet the criteria for multiple sclerosis even if they are very likely to develop it ${ }^{1}$. To our knowledge, no previous study has been carried out using USPIO on CIS patients. The overall challenge is to propose a new clinical and data processing paradigm in order to study different forms of MS at the onset and to classify them, leading to a better understanding of the early pathogenic mechanisms in MS, and allowing suitable therapeutic strategies at an early stage. To do so, we employ an unsupervised clustering method for discovering spatio-temporal patterns in CIS patients, using MRI volumes enhanced by USPIO and by Gd. The focus is on the longitudinal evolution of lesions enhanced by different contrast agents. The validation of these clusters is given by a statistical analysis, and a correlation with future TLL (enhanced and unenhanced lesions) by 15 months follow up. The prediction of TLL is relevant since it correlates with long term disabilities ${ }^{5,6}$.

\section{METHODS}

The proposed approach involved 25 CIS patients from different centers, which were assessed RRMS by follow-up. The MRI volumes were acquired by different hospitals using either a Verio 3T Siemens or an Achieva 3T Philips scanner according to a multi-center protocol. The volumes are isotropic at millimetric scale on both scanners. For each patient, at $m$ time points, we acquired different MRI volumes with an interval of three months: a T1 Gd-enhanced, a T1 USPIO-enhanced, and a T2. The USPIO-enhanced MRI were acquired after 24 hours delay to let the macrophages go in the inflammatory area, though the intravenous injection of USPIO was done on the same day of the Gd-enhanced acquisition. Gd and USPIO do not study the same phenomenon: Gd studies the blood brain barrier breakdown whereas USPIO studies the inflammatory process related to macrophages. The follow-up TLLs are assessed on T2 volumes after 15 months from the last time point $m$. A trained technician performed the manual delineation of the enhanced lesions on $\mathrm{T} 1$ and of all the lesions on $\mathrm{T} 2$ volumes. The following subsections describe the representation of lesions in time and the clustering.

\subsection{Lesion patterns features}

Once the enhanced lesions are manually delineated for all $m$ time points (two in our case), they are aligned according to their position respectively for each time point. Several features have been considered to represent the shape evolution of the lesions, such as the volume of each lesion, cell-inspired behavior ${ }^{7}$, or more advanced shape descriptor such as the Laplace-Beltrami operator ${ }^{8}$, the volume features seems to be a too oversimplified description of the shape.

In the end, a tensor-like representation combined with a hollowness index was used. Figure 1(a) depicts the process for a single lesion, and Figure 1(b) depicts an example of Gd-enhanced lesion pattern for different time-points. Practically, the lesions are represented as 3D spatial tensors and by an hollowness index $h_{i}$ (see equation (1)). This representation is also chosen because $75 \%$ of the lesions have ellipsoidal shapes or can be well approximated by an ellipsoid. The remaining $25 \%$ have a ringing shape similar to a torus ${ }^{9}$. It can be demonstrated that a tensor-like representation of a torus produces the same eigenvectors as if the central holes would have been filled but slightly larger eigenvalues. To uniform the representation, the ring-like shape has been considered as filled and the hollowness is measured and concatenated to this information. We refer to the final section for further discussion about this aspect. The hollowness is given by the ratio

$$
h_{i}=\frac{A_{\text {delineated }_{i}}}{A_{\text {full }_{i}}}
$$

where $A_{\text {delineated }}$ is the lesion area directly obtained by the manual segmentation, and $A_{\text {full }}$ is the same area with filled holes, this ratio should be 1 if there is no hole and becoming smaller than 1 if a hole is present. The ordered eigenvalues of these 3D tensors give a rotationally invariant feature representation: For each lesion at each time point, the coordinates of the $n$ voxels belonging to a specific lesion are collected in the data matrix $\boldsymbol{X}=\left[\left(x_{1}-\mu_{x}\right),\left(y_{1}-\mu_{y}\right),\left(z_{1}-\mu_{z}\right)\left|\left(x_{2}-\mu_{x}\right),\left(y_{2}-\mu_{y}\right),\left(z_{2}-\mu_{z}\right)\right| \ldots \mid\left(x_{n}-\mu_{x}\right),\left(y_{n}-\mu_{y}\right),\left(z_{n}-\mu_{z}\right)\right]$ regardless of the intensity of the voxels. Here, $\mu_{x}, \mu_{y}$ and $\mu_{z}$ are the mean of the coordinates for the $n$ voxels. These values 
are used for defining a covariance matrix $\boldsymbol{\Sigma}=\frac{1}{n-1} \boldsymbol{X} \boldsymbol{X}^{T}$, which can be decomposed in its eigenvalues $\boldsymbol{\Lambda}$ and eigenvectors $\boldsymbol{V}$ :

$$
\boldsymbol{\Sigma}=\boldsymbol{V} \boldsymbol{\Lambda} \boldsymbol{V}^{T}
$$

The eigenvectors are neglected since they represent the main directions of the lesions while the eigenvalues can represent the size of the lesions. All the three eigenvalues $\lambda$ contained in the diagonal of $\boldsymbol{\Lambda}$ and the hollowness index for all $m$ time points are the features representing the temporal evolution of the lesions. This means that for each single lesion, there is a feature vector $\boldsymbol{f}_{\boldsymbol{G} \boldsymbol{d}}$ and $\boldsymbol{f}_{\boldsymbol{U} \boldsymbol{S P I O}}$ given by

$$
f=\left[\lambda_{01}, \lambda_{02}, \lambda_{03}, h_{0}, \lambda_{11}, \lambda_{12}, \lambda_{13}, h_{1}\right],
$$

containing the values respectively for the MRI volume Gd-enhanced and USPIO-enhanced, in decreasing order for each time point to be orientation independent, with $\lambda_{i 1} \geq \lambda_{i 2} \geq \lambda_{i 3}$ for each of the $m$ time point. Once the lesions are represented for both contrast agent, the vectors $\boldsymbol{f}_{\boldsymbol{G} \boldsymbol{d}}$ and $\boldsymbol{f}_{\boldsymbol{U} \boldsymbol{S P I O}}$ of each lesion are concatenated in

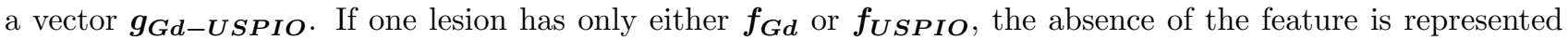
by zeros. The same lesion present in different time points $m$, is aligned to the other time representation of itself. This is performed registering all the manual delineation volume for the same patient to the volume at the first time point. If there is an overlap between the lesion of the different time point, then they are assessed automatically to be the same lesion and collected into the same $\boldsymbol{g}$ vector.

\subsection{Patient features}

The patient feature vector is given by all the cardinalities of the lesion pattern clusters $n_{C_{i}}$ present in a patient as

$$
\boldsymbol{q}=\left[n_{C_{1}}, n_{C_{2}}, n_{C_{3}}\right]
$$

e.g. $n_{C_{i}}=2$ if a lesion pattern is present twice or $n_{C_{i}}=0$ if it is not present in a specific patient. The following subsection describes how the lesion and final patient clusters are obtained.

\subsection{Clustering the lesion evolutions}

The proposed framework is based on a two layers classification. Initially lesion patterns are identified, then the discovered patterns are used to identify group of patients. These two steps are performed by the same unsupervised clustering algorithm using the output of the first layer as the input of the second, i.e. the detected lesion patterns $q$ are used as features for a second layer clustering at the patient level. Well-known unsupervised clustering algorithm are K-means, hierarchical clustering and $\mathrm{GMM}^{10}$. These approaches generally work, but they tend to fail when the nature of the data has a complicated structure or the cluster different sizes. Another possibility is to use spectral clustering ${ }^{11}$, which is based on the point-to-point similarity matrix instead of the estimate of an explicit data model. Hence, spectral clustering is adopted as clustering algorithm in the proposed framework.

Even with spectral clustering the first hurdle is the choice of parameters such as the cluster number. Since we are investigating the existence of possible patterns, we have no prior knowledge of the number of clusters and we rely on some validity indices. Many validity indices have been proposed, the reader can refer to ${ }^{12}$ for a comparison of some of them. The choice of the index depends on the nature of the data, on the subsequent used algorithm and on the number of available samples. We investigated the use of spectral-clustering and we estimated the cluster number with the algorithm proposed by Manor and Perona ${ }^{11}$ related to spectral clustering.

\subsection{Patient classification}

Once the clustering of the lesions patterns is constructed, the detected $q$ vectors representing the number of each detected lesion-based classes can be used to identify specific patients. Although the aim is not to predict TLL, the correlation between lesion patterns in patients and TLL is given to relate the patient classification to their disease severity aiming at the prediction of the most severe cases.

A regression model ${ }^{13}$ was used to examine the correlation between the number of detected lesion patterns as described in section 2.1- and the future lesion load:

$$
T L L=\beta_{0}+\beta_{1}\left(n_{C_{1}}\right)+\beta_{2}\left(n_{C_{2}}\right)+\beta_{3}\left(n_{C_{3}}\right),
$$




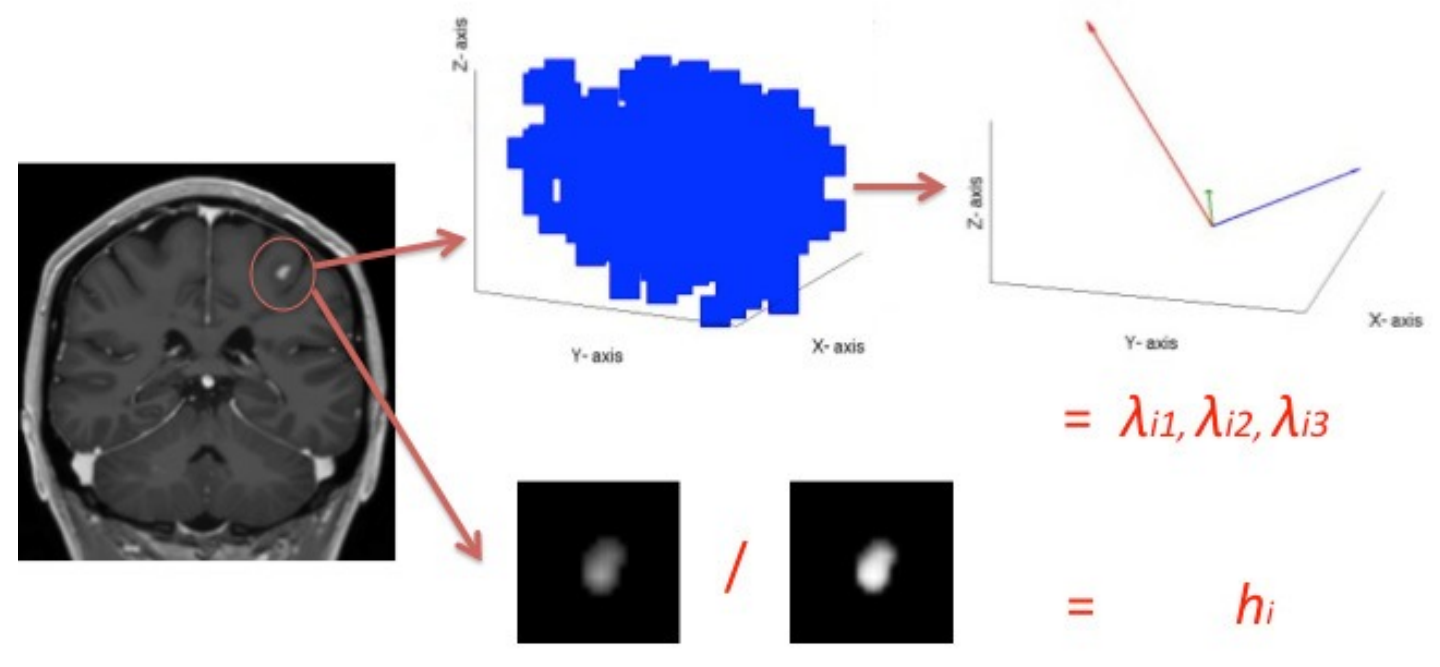

(a)
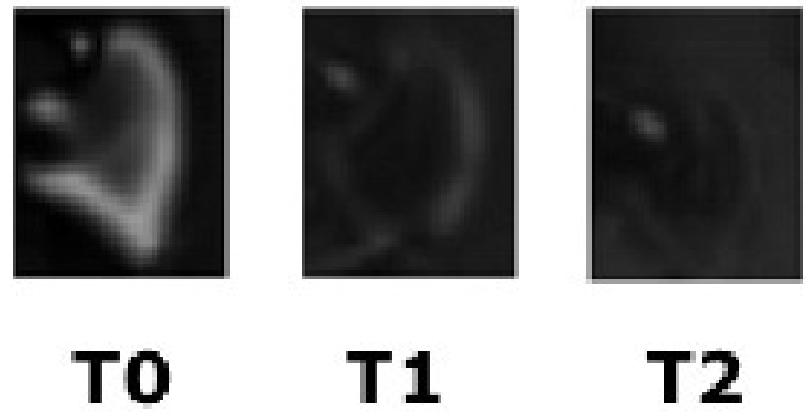

(b)

Figure 1. (a)The feature extraction process for a single time point. First all the lesions are delineated, then all the identified voxels are considered for computing the hallow index $h$ and for' building the covariance matrix and finally the eigenvalues are obtained from this covariance matrix. The process is repeated for all time points and the lesions which match in the different time point (b) are ordered in the same feature vector. 
the coefficient $\beta_{0}$ can be neglected because by construction of the model there will be at least one parameter $n_{C_{i}}$ non zero. The model examines the influence of the detected number of lesion patterns, since it is believed that some lesion patterns are more indicative of the severity of the disease. The parameters $\beta_{i}$ were estimated minimizing the Least-Squares linear regression given by $\sum\|\boldsymbol{Q} \boldsymbol{B}-\boldsymbol{T}\|^{2}$, where $\boldsymbol{B}$ is the vector containing all the $\beta_{i}, \boldsymbol{Q}$ is the matrix containing all the number of patterns per patient $q$ and $\boldsymbol{T}$ is vector containing the relative total lesion load.

\section{RESULTS}

Comparing the enhancement for both contrast agents for the same lesions, it is noted that the USPIO-enhanced lesions show a different behavior than the Gd-lesions and are often visible as a mild ringing around the Gd-lesions as depicted in Figure 2. The lesions highlighted by USPIO are highlighted by Gd as well. Our pipeline results

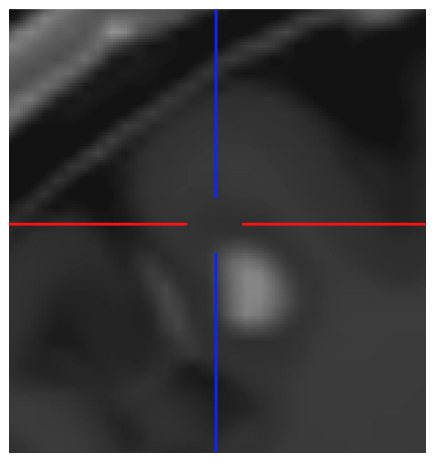

(a)

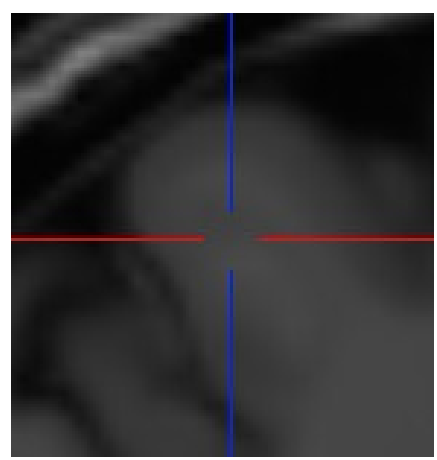

(b)

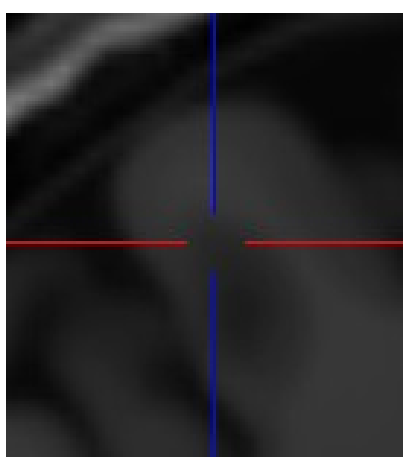

(c)

Figure 2. The same lesion at the same time point: (a) Gd, (b) USPIO and (c) pre-contrast.

highlight that the same patient can have multiple lesions belonging to different lesion clusters $C_{i}, C_{1}$ is the less specific while the other two are more specific:

- $C_{1}$ comprises lesions of different dimensions (small, average, big) appearing at the first time point and then disappearing, and generally only Gd.

- $C_{2}$ includes relatively average and big lesions present at both the first two time points, and with co-presence of USPIO and Gd, where the Gd-enhanced lesions are focal and the USPIO-enhanced lesions have a ringing behavior.

- $C_{3}$ comprises relatively average lesions present mainly at the first time point where the USPIO and Gd lesion were both non-focal (ringing or non complete focal) and approximatively of the same size..

The statistical significance is assessed computing the cluster separation ${ }^{14}$, which is defined as the average distance between each cluster centroids and the other centroids. Rank analysis over 1000000 resampled permutations of cluster separation led to the p-values $<0.0001$.

The goodness of a regression model can be assessed through the $R^{2}$ coefficient (also known as Coefficient of determination $)^{13}$. The $R^{2}$ coefficient measures of how well the regression line approximates the real data points. An $R^{2}$ of 1.0 indicates that the regression line perfectly fits the data. The most general definition of the coefficient is

$$
R^{2}=1-\frac{S S_{\text {err }}}{S S_{\text {err }}+S S_{\text {reg }}},
$$

where $S S_{\text {err }}$ is the the squared sum difference between the predicted value $\hat{\boldsymbol{T}}$ and the reference $\boldsymbol{T} . S S_{\text {reg }}$ is the squared sum difference between the predicted value and the mean value of $\boldsymbol{T}$. Also this measurement is repeated in a leave one patient out cross-validation manner.The mean and variance of all the obtained $R^{2}$ coefficients for the features $\boldsymbol{g}_{\boldsymbol{G} \boldsymbol{d}-\boldsymbol{U} \boldsymbol{S P I O}}$ was $0.68 \pm 0.19$. 


\begin{tabular}{|c||c||c||}
\hline Patient ID & lesion clusters $($ and multiplicity) & TLL in $\mathrm{cm}^{3}$ at $m_{18}$ \\
\hline 6 & $C_{2}(\times 1), C_{3}(\times 2), C_{1}(\times 37)$ & 28.9 \\
\hline 11 & $C_{1}(\times 1)$ & 9.70 \\
\hline 9 & $C_{2}(\times 1)$ & 6.72 \\
\hline 10 & $C_{2}(\times 1), C_{1}(\times 9)$ & 5.70 \\
\hline 16 & $C_{3}(\times 1)$ & 4.70 \\
\hline 24 & $C_{2}(\times 1), C_{1}(\times 2)$ & 4.63 \\
\hline 4 & $C_{1}(\times 2)$ & 3.29 \\
\hline 13 & $C_{1}(\times 2)$ & 3.50 \\
\hline 21 & $C_{1}(\times 4)$ & 3.40 \\
\hline 18 & $C_{1}(\times 2)$ & 3.32 \\
\hline 25 & $C_{1}(\times 6)$ & 2.10 \\
\hline 12 & $C_{1}(\times 9)$ & 2.10 \\
\hline 7 & $C_{1}(\times 1)$ & 1.90 \\
\hline 2 & $C_{1}(\times 1)$ & 1.73 \\
\hline 19 & $C_{1}(\times 2)$ & 1.70 \\
\hline 5 & $C_{1}(\times 4)$ & 1.27 \\
\hline 22 & $C_{1}(\times 1)$ & 1.18 \\
\hline 1 & $C_{1}(\times 1)$ & 1.18 \\
\hline 17 & no active lesions & 1.14 \\
\hline 8 & no active lesions in the first 2 time points & 0.98 \\
\hline 3 & no active lesions in the first 2 time points & 0.68 \\
\hline 15 & no active lesions in the first 2 time points & 0.54 \\
\hline 20 & no active lesions in the first 2 time points & 0.49 \\
\hline 23 & no active lesions in the first 2 time points & 0.29 \\
\hline 14 & & \\
\hline
\end{tabular}

Table 1. List of patients with the relative detected patterns, and TLL in $\mathrm{cm}^{3}$ by follow-up (15 months after the $\mathrm{m}_{3}$ ). The colors highlights the different group of patients. The patients highlighted in red have at least one lesion pattern belonging either to the cluster $C_{2}$ or to the cluster $C_{3}$, which it is considered an indication of severe condition. The patients highlighted in blue have only one lesion pattern $C_{1}$, this is the mildest condition which is clearly related to an overall moderate TLL. The patients highlighted in green are the patients which are excluded from the clustering since they do not present active lesions at the first two time points. The other cases are simply colored in black. It was noted that except patient 11 and 24, all patients correlate with their relative TLL. The lesion of patient 9 to be correctly clustered required a tuning of the neighborhood parameter for the graph construction of the spectral clustering, while the other lesions were less sensitive to this parameter.

Table 1 shows the heterogenous distribution of patterns automatically obtained, which correlates with the future total lesion load (second layer), only patient 11 and 24 seem not correlating properly. The lesion of patient 9 to be correctly clustered required a tuning of the neighborhood parameter for the graph construction of the spectral clustering, while the other lesions were less sensitive to this parameter. The lesion load is reported as unnormalized measure in $\mathrm{cm}^{3}$. The patients are assigned by the algorithm to groups which can represent different degrees of disease severity.

\section{NEW OR BREAKTHROUGH WORK TO BE PRESENTED}

A novel paradigm for spatio-temporal analysis of MS lesions has been proposed. It has been applied to a novel contrast agent USPIO that appears to give complementary information compared to the traditional Gd. We devised a two layers classification, first lesion patterns are discovered and then patients are grouped according to the lesion patterns. This final patient classification - performed at the very early stages- correlates with the future TLL after 15 months from the last used time point, this might indicate an even further evolution ${ }^{5}$. This finding could lead to early diagnosis and more suitable treatments. 


\section{CONCLUSIONS}

The framework is able to classify lesions and MS patients from the very early stages (2 first MR scans), this classification seems to be well aligned to the TLL by follow-up, which is so far the only image-based figure that can potentially correlate to future evolution of the pathology ${ }^{5,6}$. A further cluster is given by the lesions which are not detected since they do not absorb any contrast agent at the first two time points. USPIO-enhanced lesions show different behaviors than Gd-enhanced lesions which are more pronounced in some lesions rather than others correlating with future lesion loads. This leads to the hypothesis that USPIO can help to mark lesions with high risk to decline to a more severe course. Although as suggested in, ${ }^{9}$ USPIO could also highlight healing process, the presence of either lesion pattern $C_{2}$ or $C_{3}$ is a sign of intense activity.

In fact, this characterization suggests as well that belonging to a specific group of patients has an incidence on the future evolution of the disease. Although promising, the predictive value of USPIO needs to be validated on larger populations.

Lesion segmentation can be automated with a segmentation method ${ }^{15}$. The tensor-like representation for lesions is motivated by the rotationally invariant eigenvalues and the analogy to PCA. This representation can oversimplify the complexity of the lesion enhancement even if still informative. Using the eigenvalues of the Laplace-Beltrami operator ${ }^{8}$ as features can solve the issue of the hole in the ringing lesions. However, preliminary experiments showed that this method would require a very high dimensional representation which is unfeasible with the current dataset. Although, dimensionality reduction has been recently investigated with regard to the Laplace-Beltrami operator ${ }^{16}$, a correct feature selection with the current settings does not seem appropriate.

Future works are also given by different validity indices for clustering and by the inclusion of other MRI quantitative measurements (such as Magnetization Transfer, Diffusion MRI or relaxometry), and by comparing the classification to the clinical data available. In summary, the framework can predict future TLL and can provide an additional classification of the different type of lesions across patients that an actual lesion load cannot provide.

\section{REFERENCES}

[1] Leray, E., Yaouanq, J., Page, E. L., Coustans, M., Laplaud, D., Oger, J., and Edan, G., "Evidence for a two-stage disability progression in multiple sclerosis," Brain 133, 1900-1913 (2010).

[2] Lucchinetti, C., Brueck, W., Parisi, J., Scheithauer, B., Rodriguez, M., and Lassman, H., "Heterogeneity of multiple sclerosis lesions: Implications for the pathogenesis of demyelination," Annals of Neurology 47, 707-717 (2000).

[3] Bitsch, A., Schuchardt, J., S.Bunkowski, Kuhlmann, T., and Brueck, W., "Acute axonal injury in multiple sclerosis," Brain 123, 1174-1183 (2000).

[4] Dousset, V., B.Doche, Petry, K., Brochet, B., Delalande, C., and Caille, J., "Correlation between clinical status and macrophage activity imaging in the central nervous system of rats," Academic Radiology $\mathbf{9}, 156-9$ (2002).

[5] Brex, P., Ciccarelli, O., O'Riordan, J., Sailer, M., Thompson, A., and Miller, D., "A longitudinal study of abnormalities on MRI and disability from multiple sclerosis," New England Journal of Medicine 346, 158-164 (2002).

[6] Filippi, M., Horsfield, M., Morrissey, S., MacManus, D., Rudge, P., McDonald, W., and Miller, D., "Quantitative brain lesion load predicts the course of CIS," Neurology 44, 635-641 (1994).

[7] Haermae, V., Virtanen, J., Maekelae, R., Happonen, A., Mpindi, J., Knuuttila, M., Kohonen, P., Loetjoenen, J., Kallioniemi, O., and Nees, M., "A comprehensive panel of three-dimensional models for studies of prostate cancer growth, invasion and drug responses," PLoS One 5, 245-267 (2010).

[8] Reuter, M., Wolter, F., and Peinecke, N., "Laplace-Beltrami spectra as shape-dna of surfaces and solids," CAD 38, 342-366 (2006).

[9] Vellinga, M., Oude-Engberink, R., Seewann, A., Pouwels, P., Wattjes, M., van-der Pol, S., Pering, C., Polman, C., de Vries, H., Geurts, J., and Barkhof, F., "Pluriformity of inflammation in multiple sclerosis shown by ultra-small iron oxide particle enhancement," Brain 131, 800-807 (2008).

[10] Duda, R., Hart, P., and Stork, D., [Pattern Classification], Wiley (2000). 
[11] Zelnik-Manor, L. and Perona, P., "Self-tuning spectral clustering," in [NIPS], 17 (2004).

[12] Maulik, U. and Bandyopadhyay, S., "Performance evaluation of clustering algorithms," IEEE PAMI 24, 1650-1654 (2002).

[13] Steel, R. and Torrie, J., [Principles and Procedures of Statistics], vol. 70, New York: McGraw-Hill (1960).

[14] Gokcay, E. and Principe, J., "Information theoretic clustering," IEEE PAMI 24, 158-71 (2002).

[15] Karimaghaloo, Z., Shah, M., Francis, S., Arnold, D., Collins, D., and Arbel, T., "Automatic detection of Gd-enhancing multiple sclerosis lesions in brain MRI using conditional random fields," IEEE TMI 31, 1181-1194 (2012).

[16] Bernardis, E., Konukoglu, E., Ou, Y., Metaxas, D., Desjardins, B., and Pohl, K. M., "Temporal shape analysis via the spectral signature," in [15th International Conference on Medical Image Computing and Computer Assisted Intervention], (2012). 